\title{
Optimización del sistema de tratamiento fisicoquímico de una estación depuradora de aguas residuales de bebidas
} gaseosas

\begin{abstract}
RESUMEN
El presente trabajo permite resolver un grave problema económico-ambiental que afecta a la comunidad al tener unidades de tratamiento con bajo niveles de eficiencia y altos costos de operación por la adición en exceso de reactivos. Mediante la investigación aplicada y experimental, se realizó un diagnóstico para determinar los requerimientos de mantenimiento, la identificación de las concentraciones y el lugar exacto en donde se adicionan las soluciones que demanda el agua residual. Los resultados alcanzados en las pruebas establecen que la dosificación ideal de sulfato de aluminio fue de 250 ppm para la maximización de la eficiencia de remoción de la contaminación en la unidad de tratamiento en un valor mayor al $90 \%$.
\end{abstract}

Palabras-claves: Contaminación; caracterización; aguas residuales; remoción; maximización.

\section{INTRODUCCIÓN}

Las empresas que están conscientes de la necesidad de una administración responsable de los recursos naturales para la sostenibilidad de sus actividades manejan, de manera responsable, todos sus movimientos, minimizando aspectos ambientales negativos que genera la producción industrial hacia su entorno. Además, han sido muy cuidadosos en la aplicación de los conceptos de producción más limpia, generando cambios en sus procesos productivos con la finalidad de evitar el incremento de la contaminación en las fuentes de agua, debido a que «la preservación del medio ambiente, en especial del hídrico, es hoy en día no solo objeto de una fuerte demanda social, sino un mandato legal y la base de una economía sostenible» (Trapote, 2016, p. 37).

En la actualidad, las empresas del sector industrial de Guayaquil (Ecuador), que poseen en sus instalaciones sistemas de tratamiento para la depuración de sus aguas residuales industriales previa a la descarga hacia las fuentes de agua, no están operando de manera eficiente, pues generan un alto malestar interno a los accionistas en virtud de haber realizado una alta inversión para proteger la calidad de las fuentes de agua, mientras que esta no cumple su propósito y, por lo tanto, deben solventar costos de una operación inútil.

Al no remover la contaminación, se generan descargas de agua residual que presentan concentraciones en los parámetros de control que sobrepasan los valores establecidos como límite máximo permisible, lo que expone a la empresa a sanciones económicas por parte de la autoridad ambiental de aplicación responsable, así como a la remediación por la afectación ambiental.

1 Ingeniero químico y M. C. en Ingeniería Ambiental por la Universidad de Guayaquil. Actualmente, es docente de la Universidad Politécnica Salesiana y director de proyectos medioambientales en la consultora ambiental Sotecma (Guayaquil).

E-mail: vordonezr@ups.edu.ec

2 Ingeniera química y M. C. en Ingeniería Ambiental por la Universidad de Guayaquil. Además, es diplomada en Manejo Ambiental por la Escuela Superior Politécnica del Litoral (Guayaquil). Actualmente, es docente de la Universidad Politécnica Salesiana y coordinadora de proyectos medioambientales en la consultora ambiental Sotecma (Guayaquil).

E-mail: cpalaciosl@ups.edu.ec

3 Ingeniero mecánico por la Escuela Superior Politécnica del Litoral y M. C. en Educación Superior por la Universidad de Guayaquil. Actualmente, es docente en la Universidad Politécnica Salesiana (Guayaquil).

E-mail: alopez@ups.edu.ec

4 Ingeniero químico y M. C. en Educación Superior por la Universidad de Guayaquil. Actualmente, es docente en la Universidad Politécnica Salesiana (Guayaquil).

E-mail: iesuarez@ups.edu.ec 
De acuerdo con lo indicado, se realizó un trabajo de investigación aplicado con el fin de optimizar la unidad de tratamiento fisicoquímico ya existente, proceso que ues una alternativa viable, eficiente y económica» (Aragonés et al., 2009), mediante la identificación de la dosis de insumos requerida y maximizando la eficiencia de remoción de la contaminación, lo cual es muy viable en función de que «la predicción de la dosis óptima de coagulante es un factor crucial» (Bouyer et al., 2005). Se estima que el $80 \%$ de las enfermedades existentes en países en vías de desarrollo son causadas por un abastecimiento de agua deficiente (World Health Organization, 2012).

\section{METODOLOGÍA}

En esta investigación utilizamos un estudio aplicado para cumplir con el objetivo propuesto, el cual es la optimización de la planta de tratamiento de aguas residuales. La investigación aplicada, también denominada utilitaria, consiste en un análisis básico y busca la aplicación, el empleo y las repercusiones prácticas de resultados en forma inmediata o mediata a través de la transformación de una realidad actual. A este tipo de investigaciones le interesa la aplicación inmediata sobre una realidad circunstancial antes que el desarrollo de alguna teoría de va- lor universal; la mayoría de las investigaciones son de esta naturaleza (Baena, 2003).

La planta de tratamiento de aguas residuales de bebidas gaseosas fue el lugar para esta investigación, por lo que las actividades que se realizaron en este escenario se convirtieron en un estudio de campo. Asimismo, también se realizó un trabajo experimental de laboratorio que permitió evaluar de manera cuantitativa desde el inicio hasta la optimización de la planta de tratamiento de aguas residuales. Estas actividades fueron estrechamente ligadas con el estudio de campo y la investigación aplicada, y se encuentran establecidas en la tabla 1.

\section{RESULTADOS}

Los resultados son expuestos en este artículo, considerando las siguientes fases: antes de la optimización, durante la optimización y después de la optimización.

\section{Resultados antes de la optimización}

Para este ítem, se consideraron datos históricos como antecedentes, todos los datos y resultados obtenidos durante el periodo 2016, antes de realizar el diagnóstico de la planta de tratamiento y su respectiva evaluación para su optimización.

Tabla 1. Metodología aplicada para la optimización.

\begin{tabular}{|c|c|c|c|}
\hline Metodología & Actividades & Recursos necesarios & Responsables \\
\hline \multirow{2}{*}{$\begin{array}{l}\text { Investigación de campo: } \\
\text { - Recopilación de información } \\
\text { - Entrevistas, reuniones } \\
\text { - Observaciones }\end{array}$} & $\begin{array}{l}\text { Evaluación inicial de la planta de tratamiento } \\
\text { existente }\end{array}$ & $\begin{array}{l}\text { Información existente (planos, } \\
\text { análisis, pruebas) }\end{array}$ & Investigadores \\
\hline & $\begin{array}{l}\text { Identificación de cambios requeridos a la } \\
\text { unidad para su optimización }\end{array}$ & $\begin{array}{l}\text { Reactivos } \\
\text { Equipos }\end{array}$ & $\begin{array}{l}\text { Jefe de planta } \\
\text { Investigadores } \\
\text { Operadores } \\
\end{array}$ \\
\hline \multirow{3}{*}{$\begin{array}{l}\text { Investigación experimental } \\
\text { (laboratorio) }\end{array}$} & $\begin{array}{l}\text { Caracterización de las aguas residuales } \\
\text { generadas en la evaluación inicial }\end{array}$ & \multirow{3}{*}{$\begin{array}{l}\text { Equipos de laboratorio } \\
\text { Reactivos }\end{array}$} & $\begin{array}{l}\text { Investigadores } \\
\text { Operadores }\end{array}$ \\
\hline & $\begin{array}{l}\text { Pruebas de jarra durante la investigación de } \\
\text { campo y optimización }\end{array}$ & & $\begin{array}{l}\text { Investigadores } \\
\text { Operadores }\end{array}$ \\
\hline & $\begin{array}{l}\text { Caracterización de las aguas residuales } \\
\text { generadas durante y después de la optimi- } \\
\text { zación }\end{array}$ & & $\begin{array}{l}\text { Jefe de planta } \\
\text { Investigadores }\end{array}$ \\
\hline \multirow{4}{*}{$\begin{array}{l}\text { Investigación aplicada: } \\
\text { optimización de la planta de } \\
\text { tratamiento }\end{array}$} & $\begin{array}{l}\text { Mantenimiento de equipos existentes en } \\
\text { unidad de tratamiento }\end{array}$ & Proveedores de mantenimiento & $\begin{array}{l}\text { Jefe de planta } \\
\text { Investigadores } \\
\text { Operadores } \\
\end{array}$ \\
\hline & Compra de equipos y reactivos & Asignación recursos económicos & $\begin{array}{l}\text { Jefe de planta } \\
\text { Investigadores }\end{array}$ \\
\hline & $\begin{array}{l}\text { Puesta en marcha y ejecución para optimi- } \\
\text { zación de las unidades y ajuste de proceso }\end{array}$ & \multirow{2}{*}{$\begin{array}{l}\text { Equipos de laboratorio } \\
\text { Reactivos }\end{array}$} & $\begin{array}{l}\text { Jefe de planta } \\
\text { Investigadores } \\
\text { Operadores }\end{array}$ \\
\hline & Evaluación de la unidad optimizada & & $\begin{array}{l}\text { Jefe de planta } \\
\text { Investigadores }\end{array}$ \\
\hline
\end{tabular}

Fuente: elaboración propia. 


\section{Caracterización del agua residual cruda prove- niente de la planta de tratamiento}

De acuerdo con la Clasificación Internacional Industrial Uniforme (Gobierno Autónomo Descentralizado del Municipio de Guayaquil, 2013), la elaboración de bebidas no alcohólicas y aguas minerales le corresponde el Código 1594; los parámetros de control para este tipo de industria se describen en la tabla 2.

\section{Evaluación de la eficiencia de la planta de trata- miento antes de optimizar}

En la tabla 2 se presentan los valores promedio, mínimos y máximos de algunos parámetros de control; se indica el porcentaje de remoción con el que estaba operando la planta de tratamiento; $y$, se señalan los valores máximos permisibles descritos en el Libro VI del TULSMA, Anexo 1, Tabla 09 del Ministerio del Ambiente (2015), sobre los criterios de calidad para la descarga de un efluente en un cuerpo dulce.

\section{Identificación de cambios requeridos en la uni- dad de tratamiento}

El proceso de tratamiento de aguas residuales requiere el cumplimiento de requisitos mínimos para alcanzar la remoción establecida en la teoría de purificación del agua residual industrial, y con esta finalidad se realiza una revisión para establecer los cambios que se necesitan en la planta de tratamiento fisicoquímico (Russel, 2012).

\section{Modificación del punto de adición del coagulante}

En la inspección se determinó que el punto de adición del coagulante presentaba una ubicación incorrecta, lo cual generaba una destrucción de los núcleos ya formados, porque en lugar de entrar a una mezcla lenta pasan hacia una más acelerada, por la acción de una bomba. El nuevo punto de mezcla rápida se cambió en un punto previo al floculador, con lo que se evitó la destrucción de los flocs.

\section{Cambio de entrada de agua al sedimentador}

La entrada de agua residual al sedimentador estaba dispuesta en el fondo del tanque por medio de unos tubos en forma de flauta. Se observó que el problema era que los orificios del tubo estaban ubicados hacia abajo, debido a que el agua, al ingresar con fuerza, chocaba con el sedimento que estaba sedimentado y lo resuspendía (Valencia, 2016).

Se corrigió la entrada de agua residual, se sellaron los orificios del tubo dispuestos hacia abajo y se perforaron nuevos orificios, pero esta vez dispuestos hacia los lados, con dos placas (arriba y abajo) que permitieron amortiguar el flujo de entrada para no resuspender los lodos ya sedimentados.

Modificación de la entrada de agua a unidades de filtración

Tabla 2. Parámetros de monitoreo de acuerdo con el CIIU 1594.

\begin{tabular}{|c|c|}
\hline Actividad industrial & Parámetros de monitoreo \\
\hline Elaboración de bebidas no alcohólicas y aguas minerales & Aceites y grasas, DBO, DQO, SST, pH \\
\hline
\end{tabular}

Fuente: GAD Municipio de Guayaquil, 2016.

Tabla 3. Valores promedio, mínimos y máximos de parámetros químicos analizados antes de la optimización.

\begin{tabular}{|c|c|c|c|c|c|c|}
\hline & & Promedio & Mínimo & Máximo & $\begin{array}{l}\text { TULSMA- Libro VI, } \\
\text { Anexo 1, Tabla } 09\end{array}$ & $\%$ de remoción \\
\hline \multirow{2}{*}{ pH (U-pH) } & Entrada & 4,52 & 3,84 & 5,60 & --- & \multirow{2}{*}{---} \\
\hline & Salida & 6,18 & 5,38 & 6,68 & $6-9$ & \\
\hline \multirow{2}{*}{ Sólidos suspendidos (mg/l) } & Entrada & 94,83 & 45,00 & 135,00 & ---- & \multirow{2}{*}{37,26} \\
\hline & Salida & 59,50 & 27,00 & 88,00 & 130 & \\
\hline \multirow{2}{*}{ DQO (mg O2/l) } & Entrada & 9045 & 6615 & 11487 & - & \multirow{2}{*}{25,16} \\
\hline & Salida & 6770 & 4770 & 9610 & 200 & \\
\hline \multirow{2}{*}{ DBO (mg O2/l) } & Entrada & 6572 & 3800 & 8400 & 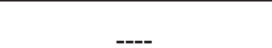 & \multirow{2}{*}{39,85} \\
\hline & Salida & 3953 & 2750 & 5396 & 100 & \\
\hline \multirow{2}{*}{ Aceites y grasas (mg/l) } & Entrada & 3,3 & 4,1 & 2,5 & --.- & \multirow{2}{*}{4,97} \\
\hline & Salida & 3,1 & 3,8 & 2,4 & 30 & \\
\hline
\end{tabular}

Fuente: elaboración propia. 
En la evaluación a los filtros se detectó que estos se encontraban taponados; el principal motivo era la baja eficiencia de remoción en el tratamiento fisicoquímico, lo que generaba la rápida sobresaturación de las capas filtrantes. Asimismo, se observó que la entrada de agua al filtro era hacia un solo punto, por lo que el agua residual no se esparcía homogéneamente en toda el área del filtro (Segura, 2009).

El primer punto fue superado con los cambios realizados en los procesos previos a la filtración. La entrada de agua residual al filtro fue cambiada para que se distribuya, homogéneamente, por medio de una hilera de diez entradas con un diámetro de cuatro pulgadas.

\section{Resultados durante la optimización}

Realizado el diagnóstico para precisar su eficiencia, se realizaron las respectivas modificaciones a las instalaciones y los procesos de dosificación de reactivos, coagulación, floculación, sedimentación y filtración para iniciar las pruebas de optimización (Marín, 2012), las cuales se detallan a continuación:

\section{Pruebas experimentales para dosificación de reactivos}

\section{Corrección del pH mediante dosificación de cal}

Se realizaron pruebas para ajustar el pH entre 6 a $7 \mathrm{U}-\mathrm{pH}$, considerando que el agua residual de la empresa se encontraba entre 3 a $5 \mathrm{U}-\mathrm{pH}$ y simulando lo que sucedía en el pozo de neutralización (Ramalho, 2015). Para ello, se dispuso diez litros de agua residual, se midió el pH inicial y se agregaron diferentes concentraciones de lechadas de cal partiendo de una solución concentradas al 10\% y una solución de trabajo al 1\%; luego se agitó el contenido y se midió el pH corregido. Se tiene como resultado que es posible corregir el $\mathrm{pH}$ entre $6,5 \mathrm{a}$ 7,5 para iniciar el proceso de coagulación y floculación con una dosis de cal entre 150 a 200 mg/l, según varíe el pH inicial (Amaya et al., 2004). Estos resultados de corrección de $\mathrm{pH}$ se encuentran en la tabla 4.

Pruebas de jarra para determinar la dosis óptima de coagulante

Se realizaron cuatro pruebas de jarra y se obtuvo como prueba de jarra óptima la que ajustó el pH a 7,5 U-pH. Como resultado, se observó una mejor formación de flóculos y sedimentación con mínimos de $5 \mathrm{mg} / \mathrm{l}$ de SST en el agua clarificada en las dosis de $250 \mathrm{mg} / \mathrm{lt}$ de sulfato de aluminio $+1 \mathrm{ppm}$ de floculante. El porcentaje de remoción alcanzó un porcentaje de $94,9 \%$. De igual manera, se mejoraron los valores de DQO desde $7540 \mathrm{mg} / \mathrm{l}$ de ARI hasta 800 ppm, que resultó la mejor dosis en esta prueba $(250+1)$. El porcentaje de remoción se mantuvo por encima de $80 \%$ en las dosificaciones de $250+1$ y $250+5$. Lo indicado se encuentra en la tabla 5.

\section{Resultados de la operación de la planta de trata- miento optimizada}

Luego de que se obtuvieron las dosis de reactivos para el tratamiento convencional fisicoquímico, se tomaron muestras al ingreso y salida de la planta de tratamiento para determinar la eficiencia de este tratamiento tras la realización de los cambios y ajustes para la optimización.

Muestreo durante la operación de la planta de tratamiento

A lo largo de cinco días, se realizó un muestreo diario, cada dos horas, durante la operación de la planta de tratamiento. Los resultados promedios se observan en la tabla 6.

\section{Resultados después de la optimización}

El siguiente análisis se realizó considerando el promedio de todos los datos existentes con el fin de

Tabla 4. Corrección de $\mathrm{pH}$ mediante lechadas de cal.

\begin{tabular}{|l|c|c|c|c|c|c|}
\hline \multicolumn{7}{|c|}{ Determinación de dosis de cal } \\
\hline \multirow{2}{*}{ Concentración cal en mg/l Pruebas } \\
\cline { 2 - 8 } & \multicolumn{3}{|c|}{ P1 } & \multicolumn{2}{c|}{ P2 } & \multicolumn{3}{c|}{ P3 } \\
\cline { 2 - 8 } & $\mathbf{p H}$ inicial & pH corregido & pH inicial & pH corregido & pH inicial & pH corregido \\
\hline 100 & 3,84 & 6,1 & 4,3 & 6,5 & 5 & 6,9 \\
\hline 150 & 3,84 & 6,8 & 4,3 & 7,2 & 5 & 7,3 \\
\hline 200 & 3,84 & 7,2 & 4,3 & 7,5 & 5 & 7,6 \\
\hline 250 & 3,84 & 7,6 & 4,3 & 7,8 & 5 & 8,1 \\
\hline
\end{tabular}

Fuente: elaboración propia. 
hacer el seguimiento y la verificación después de la optimización.

\section{Seguimiento y verificación de eficiencia}

En la tabla 7 se aprecian las concentraciones promedio del efluente tratado en la planta de tratamiento después de la optimización.

De acuerdo con los resultados de seguimiento de la tabla 7, se puede apreciar la eficiencia de la optimización del tratamiento fisicoquímico convencional que logró remover contaminantes entre el 70 y $80 \%$, cumpliendo así con los objetivos de esta investigación, que además elimina parámetros fisicoquímicos mediante la sedimentación de la mayor cantidad de sólidos en suspensión posible, para que solo circule al resto de la planta de tratamiento una mezcla mayormente líquida y homogénea.

\section{DISCUSIÓN}

La realización de los cambios identificados durante la evaluación preliminar de la unidad de tratamiento permitió optimizar el proceso de depuración.
El uso de insumos químicos en dosis inadecuadas, puntos inexactos y estados físicos inapropiados contribuía en la baja eficiencia de remoción de la contaminación.

La determinación de la dosis óptima de los reactivos de manera experimental permitió maximizar la eficiencia de remoción de la unidad de tratamiento fisicoquímico, a la vez que minimizó el consumo de los reactivos evitando la saturación de las unidades de filtración (Arboleda, 2000).

\section{CONCLUSIONES}

Se determinaron los valores de $200 \mathrm{ppm}$ de cal, 250 $\mathrm{mg} / \mathrm{lt}$ de sulfato de aluminio y $1 \mathrm{ppm}$ de polímero como dosis óptima para el tratamiento fisicoquímico de la estación depuradora de aguas residuales.

Respecto a las modificaciones realizadas para la optimización de la planta de tratamiento, se construyó un tanque reservorio junto al floculador con una línea de ingreso del efluente a tratar y el coagulante al mismo tiempo, consiguiendo una mezcla

Tabla 5. Resultados de prueba de jarra óptima.

\begin{tabular}{|c|c|c|c|c|c|c|}
\hline $\begin{array}{c}\text { Sulfato de aluminio } \\
\mathbf{m g} / \mathbf{l t}\end{array}$ & Polímero ppm & pH corregido & SST en mg/lt & \% de remoción & DQO en mg/lt & \% de remoción \\
\hline 0 & 0 & 4,3 & 98 & 0 & 7540 & 0 \\
\hline 150 & 1 & 7,5 & 21 & 78,57 & 2500 & 66,84 \\
\hline 150 & 5 & 7,5 & 18 & 81,63 & 2560 & 66,05 \\
\hline 200 & 1 & 7,5 & 15 & 84,69 & 1500 & 80,11 \\
\hline 200 & 5 & 7,5 & 10 & 89,80 & 1300 & 82,76 \\
\hline 250 & 1 & 7,5 & 5 & 94,90 & 800 & 89,39 \\
\hline 250 & 5 & 7,5 & 5 & 94,90 & 840 & 88,86 \\
\hline
\end{tabular}

Fuente: elaboración propia.

Tabla 6. Resultados promedios del agua residual durante la operación.

\begin{tabular}{|l|l|c|c|c|c|c|}
\hline \multicolumn{2}{|l|}{ Agua residual } & \multicolumn{5}{c|}{ Días } \\
\hline Parámetro & Flujo & Lunes & Martes & Miércoles & Jueves & Viernes \\
\hline \multirow{2}{*}{ pH } & Entrada & 6,72 & 6,71 & 6,71 & 6,7 & 6,7 \\
\cline { 2 - 8 } & Salida & 7,08 & 7,18 & 7,15 & 7,04 & 7,14 \\
\hline \multirow{3}{*}{ Turbidez en NTU } & Entrada & 218,4 & 317 & 864 & 514 & 529 \\
\cline { 2 - 8 } & Salida & 15,7 & 39,2 & 45,7 & 52,1 & 48,4 \\
\cline { 2 - 8 } & \% de remoción & 92,81 & 87,63 & 94,71 & 89,86 & 90,85 \\
\hline \multirow{3}{*}{ Color en UC-PT } & Entrada & 48,4 & 306 & 169 & 225 & 316 \\
\cline { 2 - 8 } & Salida & 1,8 & 5,2 & 6,1 & 5,5 & 5,5 \\
\cline { 2 - 8 } & \% de remoción & 96,28 & 98,30 & 96,39 & 97,56 & 98,26 \\
\hline \multirow{3}{*}{ DQO mg O/I } & Entrada & 9770 & 8500 & 9340 & 10030 & 9890 \\
\cline { 2 - 8 } & Salida & 340 & 285 & 470 & 920 & 1040 \\
\cline { 2 - 7 } & \% de remoción & 96,52 & 96,65 & 94,97 & 90,83 & 89,48 \\
\hline
\end{tabular}

Fuente: elaboración propia. 
Tabla 7. Seguimiento promedio de concentraciones del efluente tratado.

\begin{tabular}{|c|c|c|c|c|c|c|c|}
\hline Parámetro & Periodo & Abril & Mayo & Junio & Julio & Agosto & Septiembre \\
\hline \multirow{2}{*}{ pH } & Entrada & 4,69 & 5 & 6,265 & 6,15 & 6 & 6,2 \\
\hline & Salida & 7,15 & 6,52 & 6,59 & 7,15 & 7,4 & 7,5 \\
\hline \multirow{3}{*}{ SST } & Entrada & 218 & 384 & 193,5 & 179 & 222 & 126 \\
\hline & Salida & 25 & 59 & 43 & 24,5 & 14 & 5 \\
\hline & \% de remoción & 88,53 & 84,64 & 77,78 & 86,31 & 93,69 & 96,03 \\
\hline \multirow{3}{*}{ DQO } & Entrada & 3627 & 4627 & 5543,5 & 8590 & 5620 & 4926 \\
\hline & Salida & 470 & 470,3 & 901 & 1100 & 265 & 181 \\
\hline & \% de remoción & 87,04 & 89,84 & 83,75 & 87,19 & 95,28 & 96,33 \\
\hline \multirow{3}{*}{ DBO } & Entrada & 3280 & 2360 & 1119 & 1314 & 1209 & 1075 \\
\hline & Salida & 410,5 & 470,3 & 285 & 238 & 58 & 67 \\
\hline & $\%$ de remoción & 87,48 & 80,07 & 74,53 & 81,89 & 95,20 & 93,77 \\
\hline \multirow{3}{*}{ Aceites y grasas } & Entrada & 3,8 & 4,1 & 5,8 & 2,6 & 6,8 & 8,1 \\
\hline & Salida & 0,6 & 0,6 & 0,2 & 0,2 & 0,3 & 0,2 \\
\hline & $\%$ de remoción & 84,21 & 85,37 & 96,55 & 92,31 & 95,59 & 97,53 \\
\hline
\end{tabular}

Fuente: elaboración propia.

rápida en un tiempo de contacto de aproximadamente un minuto y permitiendo realizar el primer paso del tratamiento químico convencional. Con el cambio de las líneas de ingreso del agua a tratar en el sedimentador, se corrigió la resuspensión de los lodos formados y se logró una distribución homogénea del agua residual que ingresa a los filtros mediante la instalación de tuberías dispuestas apropiadamente.

Los resultados de los análisis realizados antes, durante y después de la optimización permitieron verificar la eficiencia de la planta de tratamiento, logrando un porcentaje de remoción mayor al $90 \%$ en las variables de sólidos suspendidos totales, aceites y grasas, DQO, DBO y el pH ajustado al rango de la descarga (Chung, 2018). Este proyecto evidencia lo importante de la interconexión de la Universidad con otros espacios de la sociedad para contribuir con su conocimiento en la mejora de la calidad de vida.

\section{REFERENCIAS BIBLIOGRÁFICAS}

[1] Amaya, W.; Cañón, Ó. y Avilés, Ó. (2004). Control de $\mathrm{pH}$ para una planta de tratamiento de aguas residuales. Ciencia e Ingeniería Neogranadina, 14(1), 86-95.

[2] Aragonés, P.; Mendoza, J.; Bes-Piá, A.; García, M. y Parra, E. (2009). Application of multicriteria decision analysis to jar-test result for chemicals selection in the physical-chemical treatment of textile wastewater. Journal of Hazardous Materials, 164(1), 288-295.
[3] Arboleda, J. (2000). Teoría y práctica de la purificación del agua. Bogotá, Colombia: McGraw-Hill.

[4] Baena, G. (2003). Metodología de la investigación. México, D. F., México: Publicaciones Culturales.

[5] Bouyer, D.; Estudié, R. y Liné, A. (2005). Experimental analysis of hidrodynamics in a jar-test. Process Safety and Environmetal Protection, 83(1), 22-30.

[6] Chung, A. (2018). Hacia un escenario futurible para la universidad pública. Conceptos, aportes y reflexiones. Lima, Perú: Fondo Editorial de la Universidad Nacional Mayor de San Marcos.

[7] Gobierno Autónomo Descentralizado del Municipio de Guayaquil (2013). Formato de instrucciones para adjuntar a los reportes semestrales. Actividades según el CIIU. F-CCA-ARI-01 versión 1.0.

[8] Marín, R. (2012). Procesos físicoquímicos en depuración de aguas. Teoría, práctica y problemas resueltos. Madrid, España: Ediciones Díaz de Santos.

[9] Ministerio del Ambiente (2015). Acuerdo Ministerial 097-A. Anexos del Libro VI del Texto Unificado de la Legislación Secundaria del Ministerio del Ambiente (TULSMA). En Norma de calidad ambiental y de descarga de efluentes: recurso agua (pp. 286-339). Quito, Ecuador: Presidencia de la República. 
[10] Ramalho, R. (2015). Tratamiento de aguas residuales. Barcelona, España: Reverté.

[11] Russel, D. (2012). Tratamiento de aguas residuales, un enfoque práctico. Barcelona, España: Reverté.

[12] Segura, J. (2009). Maquinaria para tratamiento y depuración de aguas fundamentos $y$ aplicaciones. Madrid, España: Bellisco Ediciones Técnicas y Científicas.

[13] Trapote, A. (2016). Depuración y regeneración de aguas residuales urbanas. Alicante, España: Unión de Editoriales Universitarias Españolas.
[14] Valencia, C. (2016). Aguas residuales. Una visión integral. Bogotá, Colombia: UD.

[15] World Health Organization (2012). UN-water global analysis and assessment of sanitation and drinking-water (GLAAS). The Challenge of extending and sustaninig services. Recuperado de https://apps.who.int/iris/ handle/10665/44849. 


\title{
Optimization of the physical-chemical treatment system for a carbonated water wastewater treatment plant
}

\author{
ViRgILIO ORDÓÑEZ RamíREZ \\ CARMen PALACIOS Limones ${ }^{2}$ \\ ARMANDO LÓPEZ VARGAS ${ }^{3}$ \\ IváN SuÁrez EsCOBAR ${ }^{4}$
}

\begin{abstract}
This study makes it possible to resolve a serious environmental-economic problem that affects the community, caused by treatment plants with low levels of efficiency and high operating costs due to the excessive addition of reagents. After applied and experimental research, a diagnosis was made to determine the maintenance requirements, the identification of the concentrations and the exact point where solutions needed for wastewater treatment should be added. The results obtained establish that the ideal dosage of aluminum sulfate for maximizing the contaminant removal efficiency in the treatment plant at a value greater than $90 \%$ was $250 \mathrm{ppm}$.
\end{abstract}

Keywords: Pollution; characterization; wastewater; removal; maximization.

\section{INTRODUCTION}

Companies that are aware of the need for a responsible management of natural resources for the sustainability of their activities manage, in a responsible manner, all their movements, minimizing negative environmental aspects generated by industrial production. In addition, they have been very careful in the implementation of cleaner production concepts, making changes in their production processes in order to avoid an increase of pollution in water sources, because "la preservación del medio ambiente, en especial del hídrico, es hoy en día no solo objeto de una fuerte demanda social, sino un mandato legal y la base de una economía sostenible" [the preservation of the environment, especially water, is today not only the subject of strong social demand, but also a legal mandate and the basis of a sustainable economy] (Trapote, 2016, p. 37).

Currently, industrial sector companies in Guayaquil (Ecuador), which possess treatment systems for the purification of their industrial wastewater prior to discharge into water sources, are not operating efficiently. This generates a high level of internal discomfort for shareholders, who have made a large investment to protect the quality of water sources. By not fulfilling their mission, shareholders must cover the costs of an ineffective system.

When contaminants are not removed, wastewater discharges that present concentrations that exceed the values established as the maximum permissible limit are generated. This exposes the company to economic sanctions by the responsible enforcement environmental authority, as well as to required remediation as a result of environmental impact.

1 Chemical Engineer and MSc in Environmental Engineering from the Universidad de Guayaquil. Currently working as Professor at the Universidad Politécnica Salesiana and director of environmental projects at environmental consultancy Sotecma.

E-mail: vordonezr@ups.edu.ec

2 Chemical Engineer and MSc in Environmental Engineering from the Universidad de Guayaquil. Diploma in Environmental Management from the Escuela Superior Politécnica del Litoral (Guayaquil). Currently working as Professor at the Universidad Politécnica Salesiana and environmental project coordinator at environmental consultancy Sotecma (Guayaquil). E-mail: cpalaciosi@ups.edu.ec

3 Mechanical Engineer from the Escuela Superior Politécnica del Litoral and MSc in Higher Education from the Universidad de Guayaquil. Currently working as Professor at the Universidad Politécnica Salesiana (Guayaquil).

E-mail: alopez@ups.edu.ec

4 Chemical Engineer and MSc in Higher Education from the Universidad de Guayaquil. Currently working as Professor at the Universidad Politécnica Salesiana (Guayaquil).

E-mail: iesuarez@ups.edu.ec 
In accordance with the aforementioned, an applied research study was carried out in order to optimize the existing physical-chemical treatment unit, a process that "es una alternativa viable, eficiente $y$ económica" [is a viable, efficient and economical alternative] (Aragonés et al., 2009), by identifying the dose of components needed and maximizing the pollutant removal efficiency, which is very viable since "the prediction of the optimum coagulant dose is the crucial question" (Bouyer et al., 2005). It is estimated that $80 \%$ of existing diseases in developing countries are caused by a poor water supply system (World Health Organization, 2012).

\section{METHODOLOGY}

Applied research was used to fulfill the proposed objective, which is the optimization of the wastewater treatment plant. Applied research, also called utilitarian research, consists on a basic analysis and seeks the application and practical repercussions of the results in the short or long term via the transformation of a current reality. This type of research is interested in the immediate application on a circumstantial reality rather than the development of a universal theory; most research is of this nature (Baena, 2003).

This research took place at the carbonated water wastewater treatment plant so the activities conducted in this scenario became a field study. Also, an experimental laboratory study was conducted that permitted quantitative evaluation from the beginning until the optimization of the wastewater treatment plant was achieved. These activities were closely linked to the field study and applied research and are shown in Table 1:

\section{RESULTS}

The results are presented in this article, considering the following phases: before optimization, during optimization and after optimization.

\section{Results before optimization}

Historical data were considered as antecedents, that is, all the data and results obtained during 2016, before conducting an assessment of the

Table 1. Applied methodology for optimization.

\begin{tabular}{|c|c|c|c|}
\hline Methodology & Activities & Resources needed & Responsible \\
\hline \multirow{2}{*}{$\begin{array}{l}\text { Field research: } \\
\text { - Information gathering } \\
\text { - Interviews, meetings } \\
\text { - Observation }\end{array}$} & $\begin{array}{l}\text { Initial evaluation of the existing treatment } \\
\text { plant }\end{array}$ & $\begin{array}{l}\text { Existing information (drawings, } \\
\text { analysis, tests) }\end{array}$ & Researchers \\
\hline & $\begin{array}{l}\text { Identification of changes required to the } \\
\text { unit for optimization }\end{array}$ & $\begin{array}{l}\text { Reagents } \\
\text { Equipment }\end{array}$ & $\begin{array}{l}\text { Plant manager } \\
\text { Researchers } \\
\text { Operators }\end{array}$ \\
\hline \multirow{3}{*}{ Experimental research (laboratory) } & $\begin{array}{l}\text { Characterization of wastewater generated } \\
\text { in the initial evaluation }\end{array}$ & \multirow{3}{*}{$\begin{array}{l}\text { Laboratory equipment } \\
\text { Reagents }\end{array}$} & $\begin{array}{l}\text { Researchers } \\
\text { Operators }\end{array}$ \\
\hline & $\begin{array}{l}\text { Jar testing during field research and opti- } \\
\text { mization }\end{array}$ & & $\begin{array}{l}\text { Researchers } \\
\text { Operators }\end{array}$ \\
\hline & $\begin{array}{l}\text { Characterization of wastewater generated } \\
\text { during and after optimization }\end{array}$ & & $\begin{array}{l}\text { Plant manager } \\
\text { Researchers }\end{array}$ \\
\hline \multirow{4}{*}{$\begin{array}{l}\text { Applied research: Treatment plant } \\
\text { optimization }\end{array}$} & $\begin{array}{l}\text { Maintenance of existing equipment in } \\
\text { treatment plant }\end{array}$ & Maintenance Providers & $\begin{array}{l}\text { Plant manager } \\
\text { Researchers } \\
\text { Operators }\end{array}$ \\
\hline & Purchasing equipment and reagents & Allocation of economic resources & $\begin{array}{l}\text { Plant manager } \\
\text { Researchers }\end{array}$ \\
\hline & $\begin{array}{l}\text { Launching optimization of units and pro- } \\
\text { cess adjustment }\end{array}$ & \multirow{2}{*}{$\begin{array}{l}\text { Laboratory equipment } \\
\text { Reagents }\end{array}$} & $\begin{array}{l}\text { Plant manager } \\
\text { Researchers } \\
\text { Operators }\end{array}$ \\
\hline & Evaluation of the optimized unit & & $\begin{array}{l}\text { Plant manager } \\
\text { Researchers }\end{array}$ \\
\hline
\end{tabular}

Source: Prepared by the authors. 
treatment plant and its respective evaluation for its optimization.

\section{Characterization of raw wastewater from the treatment plant}

According to the International Standard Industrial Classification (ISIC) (Gobierno Autónomo Descentralizado del Municipio de Guayaquil, 2013), the preparation of non-alcoholic beverages and mineral waters corresponds to Code 1594. The control parameters for this type of industry are described in the table 2 .

\section{Evaluation of treatment plant efficiency before optimization}

Table 2 shows the average, minimum and maximum values of some control parameters, the removal rate that the treatment plant was operating with, and the maximum permissible values described in Book $\mathrm{VI}$ of the Unified Text on Secondary Environmental Legislation (TULSMA, by its Spanish acronym), Appendix 1, Table 09 of Ministry of Environment (2015), about the quality criteria for the discharge of an effluent into a freshwater body.

\section{Identification of changes needed in the treat- ment unit}

The wastewater treatment process requires compliance with minimum requirements to achieve the removal established in the industrial wastewater treatment theory; to this end, a review is made to establish the changes that are needed in the physical-chemical treatment plant (Russel, 2012).

\section{Coagulant addition point modification}

The inspection determined that the coagulant addition point was incorrectly located, which caused the destruction of the already formed nuclei, as instead of entering into a slow mixing, they passed towards a rather rapid mixing due to the action of a pump. The new rapid mixing point was changed to a point prior to the flocculator, which prevented the destruction of the flocs.

\section{Change of water inlet to the mixer settler}

The residual water inlet to the mixer settler was arranged at the bottom of the tank by means of flute-shaped pipes. It was observed that the problem was that the holes of the tube were downwards, since the water, when entering with force, collided with the sediment that was already condensed and resuspended it (Valencia, 2016).

The residual water inlet was corrected, the holes of the tube were sealed and new holes were drilled, but this time arranged sideways, with two plates (up and down) that made it possible to dampen the inlet flow so as not to resuspend the sludge that had already settled.

Table 2. Monitoring parameters according to ISIC 1594.

\begin{tabular}{|c|c|}
\hline Industrial Activity & Monitoring Parameters \\
\hline Elaboration of non-alcoholic drinks and mineral waters & Oils and fats, BOD, COD, OSH, $\mathrm{pH}$ \\
\hline
\end{tabular}

Source: GAD Municipio de Guayaquil [Municipal Decentralized Autonomous Government of Guayaquil], 2016.

Table 3. Average, minimum and maximum values of chemical parameters analyzed before optimization.

\begin{tabular}{|c|c|c|c|c|c|c|}
\hline & & Average & Minimum & Maximum & $\begin{array}{l}\text { TULSMA- Book VI, } \\
\text { Appendix 1, Table } 09\end{array}$ & Removal rate \\
\hline \multirow{2}{*}{ pH (U-pH) } & Input & 4.52 & 3.84 & 5.60 & --- & \multirow{2}{*}{---} \\
\hline & Output & 6.18 & 5.38 & 6.68 & $6-9$ & \\
\hline \multirow{2}{*}{ Suspended solids (mg/L) } & Input & 94.83 & 45.00 & 135.00 & ---- & \multirow{2}{*}{37.26} \\
\hline & Output & 59.50 & 27.00 & 88.00 & 130 & \\
\hline \multirow{2}{*}{ DQO (mg O2/L) } & Input & 9045 & 6615 & 11487 & --- & \multirow{2}{*}{25.16} \\
\hline & Output & 6770 & 4770 & 9610 & 200 & \\
\hline \multirow{2}{*}{ DBO (mg O2/L) } & Input & 6572 & 3800 & 8400 & ---- & \multirow{2}{*}{39.85} \\
\hline & Output & 3953 & 2750 & 5396 & 100 & \\
\hline \multirow{2}{*}{ Oils and fats (mg/L) } & Input & 3.3 & 4.1 & 2.5 & --- & \multirow{2}{*}{4.97} \\
\hline & Output & 3.1 & 3.8 & 2.4 & 30 & \\
\hline
\end{tabular}

Source: Prepared by the authors.. 


\section{Modification of the entry of water to filtration units}

The evaluation of the filters detected that they were plugged. The main cause was the low removal efficiency in the physical-chemical treatment, which generated the rapid supersaturation of the filter layers. Moreover, it was observed that the water inlet to the filter was directed towards a single point, so that the residual water did not spread evenly throughout the filter area (Segura, 2009).

The first issue was overcome with the changes made in the processes prior to filtration. The residual water inlet to the filter was changed so that it is evenly distributed through a row of ten inlets with a diameter of four inches.

\section{Results during optimization}

After the diagnosis to determine the treatment plant efficiency, the facilities and dosing of reagent, coagulation, flocculation, sedimentation and filtration processes were modified accordingly to start the optimization tests (Marín, 2012), which are detailed below:

\section{Experimental tests for reagent dosing}

\section{$\mathrm{PH}$ correction by lime dosing}

Tests were carried out to adjust the $\mathrm{pH}$ between 6 and $7 \mathrm{U}-\mathrm{pH}$, considering that the company's wastewater was between 3 and $5 \mathrm{U}-\mathrm{pH}$ and simulating what was happening in the neutralization well (Ramalho, 2015). For this, ten liters of residual water were used, the initial $\mathrm{pH}$ was measured and different lime slurry concentrations were added starting from a $10 \%$ concentrated solution and a $1 \%$ working solution. Then the content was stirred and the corrected $\mathrm{pH}$ was measured. The result was that it is possible to correct the pH between 6.5 to 7.5 to start the coagulation and flocculation process with a dose of lime between 150 to $200 \mathrm{mg} / \mathrm{L}$, depending on the initial pH (Amaya et al., 2004). These pH correction results are found in the table 4 .

Jar tests to determine the optimum dose of coagulant

Four jar tests were conducted and the optimum jar test was the one that adjusted the $\mathrm{pH}$ to $7.5 \mathrm{U}-\mathrm{pH}$. As a result, a better formation of flocs and sedimentation was observed with a minimum of $5 \mathrm{mg} / \mathrm{L}$ of SST in the clarified water in doses of $250 \mathrm{mg} / \mathrm{L}$ of aluminum sulfate $+1 \mathrm{ppm}$ of flocculant. The removal rate reached $94.9 \%$. Similarly, COD values were improved from $7540 \mathrm{mg} / \mathrm{L}$ of IWW to $800 \mathrm{ppm}$, which was the best dose in this test $(250+1)$. The removal rate remained above $80 \%$ in the dosages of $250+1$ and $250+5$. This is shown in the table 5 .

\section{Results of the operation on the optimized treat- ment plant}

After the doses of reagents for the conventional physical-chemical treatment were obtained, samples

Table 4. $\mathrm{pH}$ correction by lime slurry.

\begin{tabular}{|c|c|c|c|c|c|c|}
\hline \multicolumn{7}{|c|}{ Lime dose determination } \\
\hline \multirow{2}{*}{$\begin{array}{c}\text { Lime concentration in } \\
\mathbf{m g} / \mathbf{L}\end{array}$} & \multicolumn{3}{|c|}{ Tests } \\
\cline { 2 - 7 } & \multicolumn{2}{|c|}{ T1 } & \multicolumn{2}{c|}{ T3 } \\
\cline { 2 - 8 } & Initial pH & Corrected pH & Initial pH & Corrected pH & Initial pH & Corrected pH \\
\hline 100 & 3.84 & 6.1 & 4.3 & 6.5 & 5 & 6.9 \\
\hline 150 & 3.84 & 6.8 & 4.3 & 7.2 & 5 & 7.3 \\
\hline 200 & 3.84 & 7.2 & 4.3 & 7.5 & 5 & 7.6 \\
\hline 250 & 3.84 & 7.6 & 4.3 & 7.8 & 5 & 8.1 \\
\hline
\end{tabular}

Source: Prepared by the authors..

Table 5. Optimum jar test results.

\begin{tabular}{|c|c|c|c|c|c|c|}
\hline Aluminum sulfate & Polymer ppm & pH corrected & SST in $\mathbf{~ g / L}$ & Removal rate & COD in $\mathbf{~ g / L}$ & Removal rate \\
\hline 0 & 0 & 4.3 & 98 & 0 & 7540 & 0 \\
\hline 150 & 1 & 7.5 & 21 & 78.57 & 2500 & 66.84 \\
\hline 150 & 5 & 7.5 & 18 & 81.63 & 2560 & 66.05 \\
\hline 200 & 1 & 7.5 & 15 & 84.69 & 1500 & 80.11 \\
\hline 200 & 5 & 7.5 & 10 & 89.80 & 1300 & 82.76 \\
\hline 250 & 1 & 7.5 & 5 & 94.90 & 800 & 89.39 \\
\hline 250 & 5 & 7.5 & 5 & 94.90 & 840 & 88.86 \\
\hline
\end{tabular}

Source: Prepared by the authors. 
were taken at the entrance and exit of the treatment plant to determine the efficiency of this treatment after making the changes and adjustments for optimization.

Sampling during the operation on the treatment plant

Over a five-day period, a daily sampling was carried out every two hours during the operation of the treatment plant. The average results are observed in the table 6.

\section{Results after optimization}

The following analysis was performed considering the average of all existing data in order to conduct follow-up and verification after optimization.

\section{Efficiency monitoring and verification}

Table 7 shows the average concentrations of the treated effluent in the treatment plant after optimization.

The monitoring results of Table 7 show the efficiency of the optimization of the conventional physical-chemical treatment, which managed to remove between 70 and $80 \%$ of pollutants, thus fulfilling the objectives of this research, which furthermore eliminates physical-chemical parameters through sedimentation of the greatest amount of suspended solids possible, so that only a mostly liquid and homogeneous mixture circulates to the rest of the treatment plant.

Table 6. Average results of wastewater during operation.

\begin{tabular}{|c|c|c|c|c|c|c|}
\hline \multicolumn{2}{|c|}{ Wastewater } & \multicolumn{5}{|c|}{ Days } \\
\hline Parameter & Flow & Monday & Tuesday & Wednesday & Thursday & Friday \\
\hline \multirow{2}{*}{ pH } & Input & 6.72 & 6.71 & 6.71 & 6.7 & 6.7 \\
\hline & Output & 7.08 & 7.18 & 7.15 & 7.04 & 7.14 \\
\hline \multirow{3}{*}{ Turbidity in NTU } & Input & 218.4 & 317 & 864 & 514 & 529 \\
\hline & Output & 15.7 & 39.2 & 45.7 & 52.1 & 48.4 \\
\hline & Removal rate & 92.81 & 87.63 & 94.71 & 89.86 & 90.85 \\
\hline \multirow{3}{*}{ Color in UC Pt } & Input & 48.4 & 306 & 169 & 225 & 316 \\
\hline & Output & 1.8 & 5.2 & 6.1 & 5.5 & 5.5 \\
\hline & Removal rate & 96.28 & 98.30 & 96.39 & 97.56 & 98.26 \\
\hline \multirow{3}{*}{ COD mg O $/ 2$} & Input & 9770 & 8500 & 9340 & 10030 & 9890 \\
\hline & Output & 340 & 285 & 470 & 920 & 1040 \\
\hline & Removal rate & 96.52 & 96.65 & 94.97 & 90.83 & 89.48 \\
\hline
\end{tabular}

Source: Prepared by the authors.

Table 7. Monitoring of the average concentrations of treated effluent.

\begin{tabular}{|c|c|c|c|c|c|c|c|}
\hline Parameter & Period & April & May & June & July & August & September \\
\hline \multirow{2}{*}{ pH } & Input & 4.69 & 5 & 6.265 & 6.15 & 6 & 6.2 \\
\hline & Output & 7.15 & 6.52 & 6.59 & 7.15 & 7.4 & 7.5 \\
\hline \multirow{3}{*}{ SST } & Input & 218 & 384 & 193.5 & 179 & 222 & 126 \\
\hline & Output & 25 & 59 & 43 & 24.5 & 14 & 5 \\
\hline & Removal rate & 88.53 & 84.64 & 77.78 & 86.31 & 93.69 & 96.03 \\
\hline \multirow{3}{*}{ COD } & Input & 3627 & 4627 & 5543.5 & 8590 & 5620 & 4926 \\
\hline & Output & 470 & 470.3 & 901 & 1100 & 265 & 181 \\
\hline & Removal rate & 87.04 & 89.84 & 83.75 & 87.19 & 95.28 & 96.33 \\
\hline \multirow{3}{*}{ BOD } & Input & 3280 & 2360 & 1119 & 1314 & 1209 & 1075 \\
\hline & Output & 410.5 & 470.3 & 285 & 238 & 58 & 67 \\
\hline & Removal rate & 87.48 & 80.07 & 74.53 & 81.89 & 95.20 & 93.77 \\
\hline \multirow{3}{*}{ Oils and fats } & Input & 3.8 & 4.1 & 5.8 & 2.6 & 6.8 & 8.1 \\
\hline & Output & 0.6 & 0.6 & 0.2 & 0.2 & 0.3 & 0.2 \\
\hline & Removal rate & 84.21 & 85.37 & 96.55 & 92.31 & 95.59 & 97.53 \\
\hline
\end{tabular}

Source: Prepared by the authors 


\section{DISCUSSION}

The implementation of the required changes, identified during the preliminary evaluation of the treatment unit, made it possible to optimize the treatment process.

The use of chemical inputs in inadequate doses, inaccurate points and inappropriate physical conditions contributed to the low efficiency of pollutant removal.

The experimental determination of the optimal reagents dose made it possible to maximize the removal efficiency of the physical-chemical treatment unit, while minimizing the use of reagents and avoiding saturation of the filtration units ( $\mathrm{Ar}$ boleda, 2000).

\section{CONCLUSIONS}

It was determined that $200 \mathrm{ppm}$ of lime, $250 \mathrm{mg} / \mathrm{L}$ of aluminum sulfate and $1 \mathrm{ppm}$ polymer were optimal doses for the physical-chemical treatment of the wastewater treatment plant.

Regarding the modifications made for the treatment plant optimization, a reservoir tank was built next to the flocculator with an input line for the effluent and the coagulant to be treated at the same time, achieving a rapid mixing in a contact time of approximately one minute, making possible the first step of conventional chemical treatment. By changing the inlet lines for the water to be treated in the settler, the resuspension of the sludge formed was corrected and, by installing properly disposed pipes, a homogeneous distribution of the wastewater that enters the filters was achieved.

The results of the analyses conducted before, during and after the optimization made it possible to verify the efficiency of the treatment plant, achieving a removal rate greater than $90 \%$ in the variables of total suspended solids, oils and fats, COD, BOD and a $\mathrm{pH}$ adjusted to the range of the discharge (Chung, 2018). This project demonstrates the importance of the interconnection between the university and other spaces of society to contribute knowledge in the improvement of quality of life.

\section{REFERENCES}

[1] Amaya, W.; Cañón, Ó. y Avilés, Ó. (2004). Control de $\mathrm{pH}$ para una planta de tratamiento de aguas residuales. Ciencia e Ingeniería Neogranadina, 14(1), 86-95.
[2] Aragonés, P.; Mendoza, J.; Bes-Piá, A.; García, M. y Parra, E. (2009). Application of multicriteria decision analysis to jar-test result for chemicals selection in the physical-chemical treatment of textile wastewater. Journal of Hazardous Materials, 164(1), 288-295.

[3] Arboleda, J. (2000). Teoría y práctica de la purificación del agua. Bogotá, Colombia: McGraw-Hill.

[4] Baena, G. (2003). Metodología de la investigación. México, D. F., México: Publicaciones Culturales.

[5] Bouyer, D.; Estudié, R. y Liné, A. (2005). Experimental analysis of hidrodynamics in a jar-test. Process Safety and Environmetal Protection, 83(1), 22-30.

[6] Chung, A. (2018). Hacia un escenario futurible para la universidad pública. Conceptos, aportes y reflexiones. Lima, Perú: Fondo Editorial de la Universidad Nacional Mayor de San Marcos.

[7] Gobierno Autónomo Descentralizado del Municipio de Guayaquil (2013). Formato de instrucciones para adjuntar a los reportes semestrales. Actividades según el CIIU. F-CCA-ARI-01 versión 1.0.

[8] Marín, R. (2012). Procesos físicoquímicos en depuración de aguas. Teoría, práctica y problemas resueltos. Madrid, España: Ediciones Díaz de Santos.

[9] Ministerio del Ambiente (2015). Acuerdo Ministerial 097-A. Anexos del Libro VI del Texto Unificado de la Legislación Secundaria del Ministerio del Ambiente (TULSMA). En Norma de calidad ambiental y de descarga de efluentes: recurso agua (pp. 286-339). Quito, Ecuador: Presidencia de la República.

[10] Ramalho, R. (2015). Tratamiento de aguas residuales. Barcelona, España: Reverté.

[11] Russel, D. (2012). Tratamiento de aguas residuales, un enfoque práctico. Barcelona, España: Reverté.

[12] Segura, J. (2009). Maquinaria para tratamiento $y$ depuración de aguas fundamentos $y$ aplicaciones. Madrid, España: Bellisco Ediciones Técnicas y Científicas.

[13] Trapote, A. (2016). Depuración y regeneración de aguas residuales urbanas. Alicante, España: Unión de Editoriales Universitarias Españolas.

[14] Valencia, C. (2016). Aguas residuales. Una visión integral. Bogotá, Colombia: UD. 
[15] World Health Organization (2012). UN-water global analysis and assessment of sanitation and drinking-water (GLAAS). The Challenge of extending and sustaninig services. Recuperado de https://apps.who.int/iris/ handle/10665/44849. 